\title{
Laboreal
}

Volume $12 \mathrm{~N}^{\circ} 1$ | 2016

Os Equipamentos de Proteção Individual (EPI):

protetores, mas nem sempre

\section{"Los equipos de protección individual (EPI): protectores, pero no siempre": presentación del dossier}

"Os equipamentos de proteção individual (EPI): Protetores, mas nem sempre" apresentação do dossier

"Les équipements de protection individuelle (EPI): protecteurs, mais pas toujours " présentation du dossier

"The personal protective equipment (PPE): protective, but not always" presentation of the thematic dossier

Francisco Duarte y Laurence Théry

\section{OpenEdition}

Journals

Edición electrónica

URL: http://journals.openedition.org/laboreal/2844

DOI: $10.4000 /$ laboreal.2844

ISSN: 1646-5237

Editor

Universidade do Porto

\section{Referencia electrónica}

Francisco Duarte y Laurence Théry, «"Los equipos de protección individual (EPI): protectores, pero no siempre": presentación del dossier », Laboreal [En línea], Volume 12 №1 | 2016, Publicado el 01 julio 2016, consultado el 24 septiembre 2020. URL : http://journals.openedition.org/laboreal/2844 ; DOI : https://doi.org/10.4000/laboreal.2844

Este documento fue generado automáticamente el 24 septiembre 2020

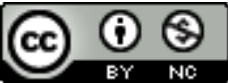

Laboreal está licenciado com uma Licença Creative Commons - Atribuição-NãoComercial 4.0 Internacional. 


\title{
"Los equipos de protección individual (EPI): protectores, pero no siempre": presentación del dossier
}

\author{
"Os equipamentos de proteção individual (EPI): Protetores, mas nem sempre" \\ apresentação do dossier \\ "Les équipements de protection individuelle (EPI): protecteurs, mais pas \\ toujours " présentation du dossier \\ "The personal protective equipment (PPE): protective, but not always" \\ presentation of the thematic dossier
}

Francisco Duarte y Laurence Théry

\section{NOTA DEL EDITOR}

http://dx.doi.org/10.15667/laborealxii0116fdes

\section{Carolina Ullilen-Marcilla}

2 En la edición de julio de la revista Laboreal, nosotros presentamos un dossier, que continuará en diciembre, que se focaliza en el uso de los Equipos de Protección Individual (EPI). Es sabido que entre los principios generales de la prevención de los riesgos laborales, estos equipos representan la última barrera frente a los riesgos a los cuales están expuestos los trabajadores.

3 Sin olvidar un artículo ya publicado en Laboreal (Garrigou, Baldi \& Dubuc, 2008), el objetivo de este dosier es discutir de manera más amplia de los diferentes aspectos muchas veces desconocidos de los EPI. En efecto, el tema de los EPI ha sido abordado principalmente desde un enfoque técnico por los químicos o especialistas de los 
materiales durante mucho tiempo. Raros estudios han abordado las dificultades durante su utilización. Esto puede conducir a un punto de vista relativamente simplista: bastaría utilizarlos para estar protegido.

4 El ángulo de ataque de este número es sacar a la luz toda la complejidad de estos objetos aparentemente simples y de sus utilizaciones. Esta complejidad concierne primero su concepción y su eficacia real que es a menudo poco evaluado. Un segundo aspecto de esta complejidad concierne su utilización en las situaciones reales. Pues, los trabajadores sienten una serie de dificultades y molestias afectando al desarrollo de sus actividades. Ahora bien, el diseño de estos equipos debe garantizar una protección óptima y perturbar lo mínimo posible, asimismo el usuario debe estar informado sobre su uso y mantenimiento apropiado afín de reducir la exposición.

5 El conjunto de los artículos convoca diferentes disciplinas - ergonomía, ciencias de la salud, ciencias jurídicas y economía, antropología, ingeniería de la prevención. Estos artículos buscarán presentar la complejidad de las formas descritas anteriormente y de manera complementaria.

6 Seis artículos forman parte de este dosier, publicado en este número de julio. Un artículo de la exposición profesional de productos químicos CMR ha sido propuesto por Fabienne Goutille, Louis Galey, Clémence Rambaud, Pierrick Pasquereau, José Marçal Jackson $\mathrm{F}^{\circ}$ y Alain Garrigou. Las medidas de prevención más frecuente se limita a los equipos de protección individual, sin embargo la reglamentación privilegia los equipos de protección colectivo. El trabajo presentado sobre la forma de un estudio de casos desde la perspectiva metodológica en ergotoxicología y también en antropología, busca articular los conocimientos sobre los peligros de los productos, las modalidades de prescripción de los EPI por la empresa y la percepción y las representaciones que tienen los trabajadores de los riesgos, los efectos en su cuerpo y la eficacia de los EPI.

7 En lo que concierne a la utilización de los EPI en el sector agrícola, Carolina Ullilen y Alain Garrigou tratan de poner en evidencia la influencia de la percepción del riesgo en el uso de estos equipos para protegerse contra los pesticidas. El estudio se realizó a una muestra de cinco viticultores, buscando comprender sus estrategias empleadas para protegerse contra los pesticidas durante la preparación de la mezcla, su aplicación en los campos agrícolas y la limpieza de los materiales agrícolas. Los resultados muestran que el nivel de formación, la experiencia adquirida con el tiempo y las diferentes creencias pueden influenciar, positiva o negativamente, en la percepción del riesgo.

8 En cuanto al diseño de los EPI, Maria Cristina Gonzaga y Cristiane Queiroz analizan el proceso de certificación de los guantes de protección en el corte manual de la caña de azúcar y la brecha entre las normas de prueba en su fabricación y la actividad a realizar, en base a investigaciones anteriores. Resulta importante reconocer la variabilidad del tamaño de las manos para la elección de los guantes y la cantidad de accidentes y enfermedades relacionadas con las manos. Asimismo, el machete considerado como la principal herramienta de trabajo ha sido modificado por los trabajadores para mejorar su rendimiento. Una serie de propuestas han sido planteadas por ellos para mejorar el diseño de los guantes y del machete, sin dejar de lado el sistema de certificación.

9 Por otra parte, el artículo de Alice Turcot y Michel Lehoux abordan el uso de los guantes antivibratorios para reducir la vibración mano-brazos. Una revisión de la literatura fue realizada en Medline (PubMed) y EBSCOhost en los años de 1970 a 2014. Un estudio de terreno con cuestionarios y entrevistas se llevó a cabo en 30 
trabajadores, a quienes se les pidió utilizar dos tipos de guantes en un corto período. Ambos equipos fueron considerados por los trabajadores como inconfortables y que no facilitaba la ejecución del trabajo. Además, la revisión de la literatura revela que la eficacia de los guantes para atenuar los niveles de vibración es baja.

Así también, otro estudio de Marcello Motta Veiga y Carlos Campelo de Albuquerque analiza la permeabilidad de los EPI contra los pesticidas por el personal sanitario para luchar contra los mosquitos Aedes aegypti. La permeabilidad es una característica de los EPI que los convierte inadecuados para su utilización, la cual fue evaluada por el método de la pipeta, calculando los porcentajes de repulsión, retención y penetración de los pesticidas en los equipos de protección. Los resultados muestran que el tipo de costura influye en la permeabilidad; y que una repulsividad inferior puede no ser suficiente para evaluar la eficacia de EPI.

11 En otro plano, la investigación de Thiara De Ângeli Porto y Mônica de Fatima tiene por objetivo comprender el autocuidado de la persona afín de proteger la salud y seguridad en los métodos de humidificación en el tratamiento de granito del estado de Espirito Santo en Brasil. Adoptando la postura de un cartógrafo, utilizando datos incluidos en un libro de registro, así como de las entrevistas con 10 trabajadores, el estudio ilustra algunos modos de usos del cuerpo durante la actividad en cuestión, poniendo en evidencia la utilización inadecuada de los EPI y la falta de conciencia de las potencialidades de los equipos de protección colectiva.

Un agradecimiento a todos los autores por contribuir con sus conocimientos y experiencias.

\section{BIBLIOGRAFÍA}

Garrigou, A., Baldi, I. \& Dubuc, P. (2008). Aportaciones de la ergotoxicología en la evaluación de la eficacia real de los EPI que deben proteger del riesgo fitosanitario: del análisis de la contaminación al proceso colectivo de alerta. Laboreal, 4, (1), 92-103.

\section{AUTORES}

\section{FRANCISCO DUARTE}

Programa de Engenharia de Produção - Universidade Federal do Rio de Janeiro - PEP/COPPE/ UFRJ, Centro de Tecnologia, Bloco G sala G209 - Cidade Universitária, 21945-970 Rio de Janeiro Brasil

duarte@pep.ufrj.br

\section{LAURENCE THÉRY}

Centre d'Ergonomie et de Sécurité du Travail de Picardie (CESTP), Agence Régionale pour l'Amélioration des Conditions de Travail (ARACT), 19, rue V. Hugo, 80000 Amiens - France l.thery@anact.fr 